\title{
Assessing the risk factors of under-five deaths in rural parts of Ethiopia
}

Yenew Alemu Mihret ( $\nabla$ yenewalemu@gmail.com )

Injibara Univresity

Research article

Keywords: Mortality, under-five mortality, rural, Ethiopia

Posted Date: April 26th, 2021

DOI: https://doi.org/10.21203/rs.2.11962/v2

License: (c) (i) This work is licensed under a Creative Commons Attribution 4.0 International License. Read Full License 


\title{
Assessing the risk factors of under-five deaths in rural parts of Ethiopia Yenew Alemu Mihret \\ Department of statistics, College of Natural and Computational Sciences, Injibara Univresity, Injibara, Ethiopia
}

Email: yenewalemu@gmail.com

\begin{abstract}
Background of the study: Under-five mortality is the likelihood for a child born alive to die between birth and fifth birth day. Mortality under the age of five has been the main problem in public health policies especially in rural parts of Ethiopia.
\end{abstract}

Objective: The objective of this study was to assess the risk factors of under-five mortality in Ethiopia using the 2011 EDHS data.

Results: Information from 8,668 women included in the study show that $64.5 \%$ of the women never experienced under-five deaths of their children. Among four possible count models considered, the ZINB regression model was selected as the most appropriate model.

Conclusion: The study revealed that mother's age first birth, breastfeeding status, wealth index, whether the mother is currently working, region and mother's level of education had statistically significant association with the number of under-five deaths in rural parts of Ethiopia.

Keyword: Mortality, under-five mortality, rural, Ethiopia

\section{Introduction}

Under-five mortality is defined as the likelihood for a child born alive to die between birth and fifth birth day. Mortality under the age of five has been the main target of public health policies and is a common indicator of mortality levels, especially in developing countries [8]. It is also viewed as an indicator of the level of development, health and socioeconomic status of the population.

Sub-Saharan Africa still has the world's highest rate of child mortality, despite reducing the number of under-five deaths by $48 \%$ since 1990. Sub-Saharan Africa is the region most affected and accounts for more than one-third of deaths of children under the age of five. In 2013, about half of global under-five deaths occurred in sub-Saharan Africa and 32 percent in South Asia. 
Around half of under-five deaths occurred in just five countries: India (21\%), Nigeria (13\%), Pakistan (6\%), Democratic Republic of the Congo (5\%) and China (4\%) [20].

Under-five death is showing a declining trend over the last decades. Numbers of under-five death rates were 166 per 1000 live births in 2000 and 88 per 1000 live births in 2011. According to the report by [6] the level of under-five mortality in rural parts of Ethiopia is 114 deaths per 1000 live births. However, the rate is still very high and requires intervention.

\section{Methods}

\subsection{Source of the data}

The source of the data for this study was the 2011 Ethiopia Demographic and Health Survey.

\subsection{Variables in the study}

The dependent variable for this study is the number of deaths of under- five deaths per mother. Based on the [15] determinants of under-five mortality for developing countries, the main predictors explored for under-five mortality are mother's age at firth birth, whether a mother is currently breastfeeding, mother's marital status, mother's level of education, whether a mother is currently working, region, household's wealth index, Source of drinking water and Toilet facility.

\subsection{Methods of data analysis}

In this study, the variable of interest is a count variable. When the response or dependent variable is a count, it is appropriate to use count regression model.

\section{Poisson regression model}

Poisson regression analysis is a technique which allows to model dependent variables that describe count data [3]. According to [19], the apparent simplicity of Poisson comes with the variance and mean of the count variable are assumed to be equal and the occurrences of the event are assumed to be independent of each other.

The Poisson regression model is given by

$$
P\left(Y_{i}=y_{i}, \mu\right)=\frac{e^{-\mu_{i} \mu_{i} y_{i}}}{y_{i} !}, \mu_{i}>0, i=1,2, \ldots n \text { and } y_{i}=0,1,2, \ldots
$$


where, $\mathrm{y}_{\mathrm{i}}$ denotes the value of an event count outcome variable occurring in a given time or exposure periods with mean parameter $\mu_{\mathrm{i}}$.

In PRM, let $\mu_{\mathrm{i}}$ as the expected number of under five-child death from the $i^{\text {th }}$ mother and the total number children ever born from the $\mathrm{i}^{\text {th }}$ mother is $\mathrm{N}_{\mathrm{i}}$. Thus the distribution of $\mathrm{Y}_{\mathrm{i}}$ can be written as:

\section{$\mathbf{Y}_{\mathbf{i}} \sim$ poisson $\left(\mathrm{N}_{\mathrm{i}} \boldsymbol{\mu}_{\mathbf{i}}\right)$}

Where, $\mathbf{N}_{\mathbf{i}}$ is the total fertility rate of $\mathrm{i}^{\text {th }}$ mother

The logarithm of the children ever born is introduced in the regression model as an offset variable [9].

\section{Negative binomial regression model}

This model is used when count data are over-dispersed (i.e when the variance exceeds the mean).

Over-disprsion, caused by heterogeneity or an excess number of zeros (or both) to some degree is inherent to most Poisson data. [11] used negative binomial regression to model over dispersed Poisson data.. The NB regression model is

$$
\mathrm{P}\left(\mathrm{y}_{\mathrm{i}} ; \mu \mathrm{i}, \alpha,\right)=\frac{\Gamma\left(\mathrm{y}_{\mathrm{i}}+1 / \alpha\right)}{\mathrm{y}_{\mathrm{i}} ! \Gamma(1 / \alpha)}\left(1+\alpha \mu_{\mathrm{i}}\right)^{\frac{-1}{\alpha}}\left(1+\frac{1}{\alpha \mu_{\mathrm{i}}}\right)^{-\mathrm{y}_{\mathrm{i}}}, \quad \mathrm{y}_{\mathrm{i}} \geq 0 \quad \text { and } \quad \alpha>0 .
$$

with mean and variance are given by

$$
\mathrm{E}\left(\mathrm{Y}_{\mathrm{i}}\right)=\mu_{i}=\exp \left(x_{i}^{T} \beta\right) \text { and } \operatorname{Var}\left(\mathrm{Y}_{\mathrm{i}}\right)=\mu_{i}\left(1+\alpha \mu_{i}\right)
$$

where, $\alpha$ shows the level of overdispersion and $\Gamma($.$) is the gamma function.$

\section{Zero- inflated Poisson regression model}

The Zero-inflated Poisson regression study the relationship between dependent and independent variable(s) when there are many zeros value in the dependent variable.

The ZIP regression model is [14],

$$
P\left(y_{i}\right)=\left\{\begin{array}{l}
\omega_{i}+\left(1-\omega_{i}\right) e^{-\mu_{i}}, \quad y_{i}=0 \\
\quad\left(1-\omega_{i}\right) \frac{e^{-\mu i_{1}} \mu_{i} y_{i}}{y_{i} !}, \quad y_{i}=1,2, \ldots, \quad 0 \leq \omega_{i} \leq 1
\end{array}\right.
$$

where, $Y_{i} \sim Z I P\left(\mu_{i}, \omega_{i}\right)$.

\section{Zero-inflated negative binomial regression model}

Zero-Inflated Negative Binomial regression is used in troubleshooting over-dispersion due to excessive zero values in the response variable. [10] used the zero-inflated negative binomial 
regression to model over-dispersed data with an excess of zeros. This regression model was given by

$$
P\left(y_{i} \mid \omega, \alpha, \mu\right)=\left\{\begin{array}{l}
\omega_{i}+\left(1-\omega_{i}\right)\left(1+\alpha \mu_{i}\right)^{\frac{-1}{\alpha}}, \quad y_{i}=0 \\
\left(1-\omega_{i}\right) \frac{\Gamma\left(y_{i}+1 / \alpha\right)}{y_{i} ! \Gamma(1 / \alpha)}\left(1+\alpha \mu_{i}\right)^{\frac{-1}{\alpha}}\left(1+\frac{1}{\alpha \mu_{i}}\right)^{-y_{i}}, \quad y_{i}>0
\end{array}\right.
$$

where, $\mu_{i}$ is the mean of the underlying negative binomial distribution, $\alpha>0$ is the over dispersion parameter and is assumed not to depend on covariates and $0 \leq \omega_{i} \leq 1$. Also the parameters $\mu_{i}$ and $\omega_{i}$ depend on vectors of covariates $x_{i}$ and $z i$, respectively.

\section{Goodness of fit tests}

The overall test statistic for likelihood ratio test was used.

The Vuong test is a non-nested test that is based on a comparison of the predicted probabilities of two models that do not nest [21]. Another selecting an appropriate models are Akaike information criteria [2] and Baysians information criteria [18] abbreviated by AIC and BIC, respectively.

\section{Result}

Information on the number of deaths of under-five children obtained from a total of 8,668 women in the rural parts of Ethiopia was studied. Among the 8,668 women, considered, 5,180 children died before the age of five. As shown in the summary Table 1, ZINB model has the smallest AIC and BIC and maximum log-likelihood, ZINB model is the most appropriate and preferred model among the four models. By using Vuong test and figure 1 also confirms that the ZINB model is the most appropriate model among four models considered.

The results in Table 2 shows that the expected number of under-five deaths for women from Amhara region was 1.15 times the expected number of under-five deaths for women in the Tigray holding all other variables in the model constant. Similarly, the expected number of under-five deaths increased by a factor of 1.17 for women living in Oromiya compared to women in Tigray. In addition, the expected number of under-five mortality for women from Benshangul-gumuz, SNNP and Gambella had increased by a factor of 1.45, 1.36 and 1.33 as compared to the expected number of under-five mortality in Tigray region, respectively. 
Based on table 2, the expected number of under-five deaths for women decreased by a factor of 0.64 in the age group 15-19, decreased by a factor of 0.50 in the age group 20 and above compared to those in the age group less than 15.

The finding of this study also revealed that the expected number of under-five mortality for mothers with primary education decreased by a factor of 0.64 as compared to those with no education. In addition, the expected number of under-five mortality for mothers with secondary and higher level of education decreased by a factor of 0.38 and 0.19 as compared to those with no education, respectively.

In this study, the factor whether mother is currently breastfeeding has a significant effect on the number of under-five deaths. The estimated number of under-five mortality for mothers who were breastfeeding is about 0.78 times lower than that for mothers who were not breastfeeding. In addition, mothers work status had a significant association with the number of under- five deaths. The expected number of under- five deaths increased by a factor of 1.09 for working mothers as compared to that for non-working mothers.

According to the findings of this study, the expected number of under-five deaths for women in the medium and rich households was 0.92 and 0.91 times the expected number of under-five deaths for women in the poor households, respectively, while holding all other variables in the model constant. As shown in Table 2, mother's marital status has a significant effect on the probability of being an excess zero. The odds of being in the zero groups are increased by a factor of 2.82 for unmarried mothers as compared to married mothers controlling for other variables in the model.

\section{Discussion}

This study was carried out to identify the risk factors of under-five mortality in rural areas of Ethiopia based on EDHS 2011 data. As the results revealed, the number of under-five mortality in rural Ethiopia has a wide variation among regions. This result is consistent with the findings by [7].

In this study, mothers' education was found to be an important socio-economic predictor of the number of under-five mortality in rural Ethiopia. Under-five mortality decreased with increased 
level of mother's education. This result is consistent with the findings by [24], [5], [1], [12-13] and [17].

The current study revealed that those children whose mother's age at first birth was below 15 years had the higher risk of dying relative to children whose mothers' age was 15 and above. A similar study in Kenya by [7] also found that mothers' age at first birth has a significant effect on infant and child mortality showing that a child born to a younger mother experienced the highest risk of dying. These findings are consistent with the findings of [1] and [17].

According to the results, mother's current breastfeeding status was a significant determinant of under-five mortality showing that children born to non-breastfeeding mother experience higher risk of mortality than children born to breastfeeding mothers. This result is consistent with the findings by [16-17].

The findings suggested that under-five child mortality risk is higher for children of poor mothers compared to children of medium and rich mothers. This finding is consistent with [16], [7], [22] and [5].

The study indicated that children born from working mothers have higher risk of mortality than non-working mothers. This finding is consistent with [5].

The results of ZINB indicated that marital status has a significant factor on the odds of being in the always zero group. The odds of being in the always zero group for unmarried mothers were higher than unmarried mothers.

\section{Conclusion}

The main objective of the study was to identify some of the factors that influence the number of infant and child mortality at rural levels. The study was based on secondary data obtained from the central statistical agency of Ethiopia. The study revealed that mother's age at first birth, whether a mother is currently breastfeeding, wealth index, whether a mother is currently working, region and mother's level of education had statistically significant effect on the number of under-five deaths in rural parts of Ethiopia. 


\section{Limitations}

The data used in this study are from the EDHS 2016. Thus, the results may not necessarily reflect the current situation of Ethiopia and Child above the age of five are not considered in this study.

\section{Declarations}

\section{Ethics approval and consent to participate}

This study used secondary data from EDHS case records and patients were not contacted. The data from the case records were handled with strong responsibility and confidentiality. The study was started after ethical clearance was obtained from Injibara University research committee and permission was taken from central statistics agency.

\section{Consent to publish}

Not applicable

\section{Availability of data and materials}

If needed the raw data in excel format for this article is available.

\section{Competing interest}

The author declares no competing interests.

\section{Funding}

The author received no specific funding for this work.

\section{Author's contributions}

Yenew Alemu conceptualized, designed, analyzed, interpreted the results and wrote the manuscript. Mekonnon Tadesse give guidance, checked the appropriateness of the modes and helped in the interpretation of the results.

\section{Acknowledgements}

I thankful to the EDHS coordinators for the data. 


\section{References}

1. Abimbola O,Adepoju AO, Akanni O and Falusi AO. Determinants of child mortality in rural Nigeria. World Rural Observations [2012;4(2):38-45]. ISSN: 1944-6543 (Print); ISSN: 19446551. http://www.sciencepub.net/rural.

2. Bozdogan H. Model selection and Akaike's information criterion (AIC): The general theory and its analytical extensions. Psychometrika. 1987 Sep 1;52(3):345-70.

3. Cameron AC, Trivedi PK. Regression analysis of count data. Cambridge university press; 2013 May 27.

4. Dalrymple ML, Hudson IL, Ford RP. Finite mixture, zero-inflated Poisson and hurdle models with application to SIDS. Computational Statistics \& Data Analysis. 2003 Jan 28;41(34):491-504.

5. Ermias D. Determinants of Child Survival Chances in Rural Ethiopia. Adama Science and Technology University, Adama, Ethiopia. Proceedings 59th ISI World Statistics Congress, 25-30 August 2013, Hong Kong (Session CPS105).

6. Demographic E. Health Survey Central Statistical Agency Addis Ababa. Ethiopia ICF International Calverton, Maryland, USA. 2011:180-6.

7. Ettarh RR, Kimani J. Determinants of under-five mortality in rural and urban Kenya. Rural \& Remote Health. 2012 Jan 1;12(1).

8. Garenne M, Gakusi E. Health transitions in sub-Saharan Africa: overview of mortality trends in children under 5 years old (1950-2000). Bulletin of the World Health Organization. 2006;84:470-8.

9. Ko MK, Sawangdee Y, Gray R, Hunchangsith P. Ecological analysis of community-level socioeconomic determinants of infant and under-five mortality in Myanmar: an analysis of the 2014 Myanmar population and housing census. Journal of Health Research. 2017;31(1):57-68.

10. Gurmu S, Trivedi PK. Excess zeros in count models for recreational trips. Journal of Business \& Economic Statistics. 1996 Oct 1;14(4):469-77.

11. Hilbe JM. Negative binomial regression. Cambridge University Press; 2011 Mar 17.

12. Wang L, Jacoby H. Environmental Determinants of Child Mortality in Rural China: A Competing Risks Approach. The World Bank; 2004 Mar 12. 
13. Van der Klaauw B, Wang L. Child mortality in rural India. World Bank Policy Research Working Paper. 2004 Apr 21(3281

14. Lambert D. Zero-inflated Poisson regression, with an application to defects in manufacturing. Technometrics. 1992 Feb 1;34(1):1-4.

15. Mosley WH, Chen LC. An analytical framework for the study of child survival in developing countries. Population and development review. 1984 Jan 1;10(0):25-45.

16. Mustafa HE, Odimegwu C. Socioeconomic determinants of infant mortality in Kenya: analysis of Kenya DHS 2003. J Humanit Soc Sci. 2008;2(8):1934-722.

17. Mani K, Dwivedi SN, Pandey RM. Determinants of under-five mortality in Rural Empowered Action Group States in India: An application of Cox frailty model. International Journal of MCH and AIDS. 2012;1(1):60.

18. Raftery AE. Choosing models for cross-classifications. American sociological review. 1986 Feb 1;51(1):145-6.

19. Sturman MC. Multiple approaches to analyzing count data in studies of individual differences: The propensity for type I errors, illustrated with the case of absenteeism prediction. Educational and Psychological Measurement. 1999 Jun;59(3):414-30.

20. You D, Hug L, Ejdemyr S, Idele P, Hogan D, Mathers C, Gerland P, New JR, Alkema L. Global, regional, and national levels and trends in under-5 mortality between 1990 and 2015, with scenario-based projections to 2030: a systematic analysis by the UN Inter-agency Group for Child Mortality Estimation. The Lancet. 2015 Dec 5;386(10010):2275-86.

21. Vuong QH. Likelihood ratio tests for model selection and non-nested hypotheses. Econometrica: Journal of the Econometric Society. 1989 Mar 1:307-33.

22. Silva P. Environmental factors and children's malnutrition in Ethiopia. The World Bank; 2005 Jan 12.

23. Wang P, Puterman ML, Cockburn I, Le N. Mixed Poisson regression models with covariate dependent rates. Biometrics. 1996 Jun 1:381-400.

24. Seyoum Y, Sharma MK. Survival Analysis of Under Five Mortality in Rural Parts of Ethiopia. International Journal of Statistics in Medical Research. 2014 Aug 5;3(3):266-81. 


\section{Appendices}

Figure 1 residual plots for estimated models

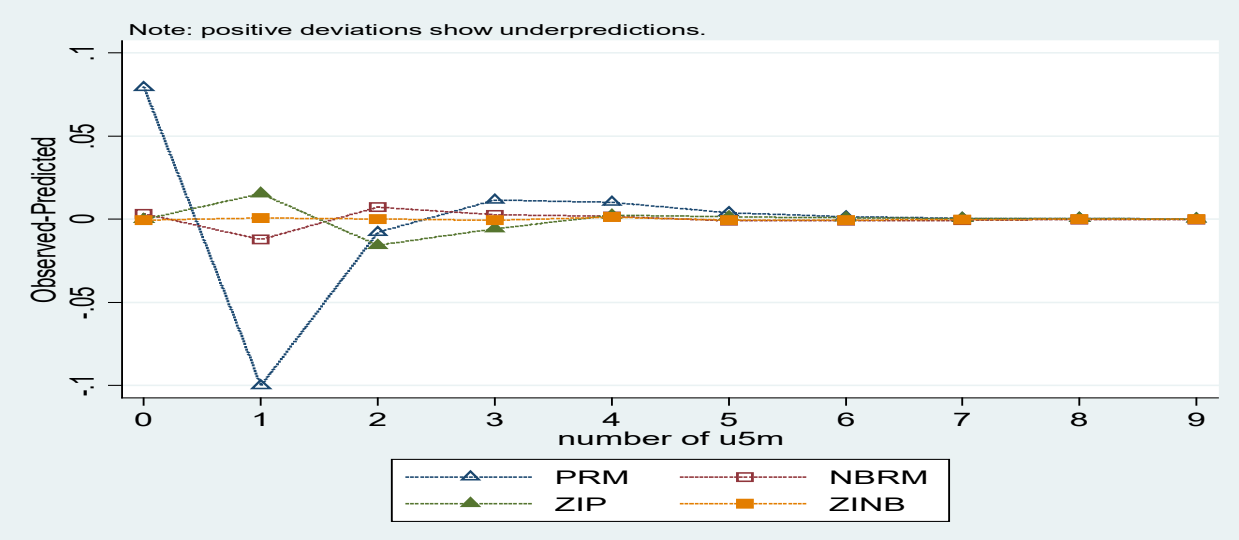

Table 1 Fit statistic of count regression models

\begin{tabular}{|c|c|c|c|c|}
\hline criteria & Poisson & NB & ZIP & ZINB \\
\hline Log-likelihood & -8412.6821 & -8238.5233 & -8253.059 & -8227.931 \\
\hline$-2 \mathrm{LL}$ & $16,825.3648$ & $16,477.0466$ & $16,506.118$ & $16,455.862$ \\
\hline AIC & 16865.36 & 16519.05 & 16548.12 & 16499.86 \\
\hline BIC & 17006.71 & 16667.46 & 16696.53 & 166555.35 \\
\hline $\begin{array}{l}\text { Likelihood } \\
\text { ratio test }\end{array}$ & $\begin{array}{l}561.03 \\
(0.000)\end{array}$ & $\begin{array}{l}405.93 \\
(0.000)\end{array}$ & $424.52(0.000)$ & $403.19(0.000)$ \\
\hline \multicolumn{2}{|l|}{ Model } & Vuong Statistic(V) & \multicolumn{2}{|c|}{ Preferred model } \\
\hline \multicolumn{2}{|l|}{ ZIP VS Poisson } & 8.16 & \multicolumn{2}{|l|}{ ZIP } \\
\hline \multicolumn{2}{|l|}{ ZINB VS NB } & 2.46 & \multicolumn{2}{|l|}{ ZINB } \\
\hline
\end{tabular}

Table 2 Estimated coefficients of ZINB regression model

\begin{tabular}{|c|r|r|r|r|rl|}
\hline variables & Coef. & Std. Err. & \multicolumn{1}{c|}{$\mathrm{z}$} & $\mathrm{P}>|\mathrm{z}|$ & \multicolumn{2}{|c|}{$[95 \% \mathrm{Cl}]$} \\
\hline Region,Tigray(ref.) & & & & & & \\
\hline Affar & 0.0416065 & 0.0724009 & 0.57 & 0.566 & -.1002966 & .1835095 \\
\hline Amhara & 0.1411311 & 0.0690014 & 2.05 & 0.041 & .0058908 & .2763715 \\
\hline Oromiya & 0.1569805 & 0.0657261 & 2.39 & 0.017 & .0281598 & .2858012 \\
\hline
\end{tabular}




\begin{tabular}{|c|c|c|c|c|c|}
\hline Somali & 0.0403798 & 0.0779589 & 0.52 & 0.604 & $-.1124168 \quad .1931763$ \\
\hline Benshangul- Gumuz & 0.3706548 & 0.0706173 & 5.25 & 0.000 & $.2322474 \quad .5090622$ \\
\hline SNNP & 0.3081286 & 0.065627 & 4.7 & 0.000 & $.179502 \quad .4367552$ \\
\hline Gambela & 0.2633426 & 0.0806256 & 3.27 & 0.001 & $\begin{array}{ll}.1053194 & .4213659\end{array}$ \\
\hline Harari & -0.0685959 & 0.0986595 & -0.7 & 0.487 & $-.261965 \quad .1247732$ \\
\hline Dire Dawa & 0.1177358 & 0.092601 & 1.27 & 0.204 & $-.0637588 \quad .2992305$ \\
\hline \multicolumn{6}{|l|}{$\begin{array}{l}\text { Mother level of education, } \\
\text { no education(ref.) }\end{array}$} \\
\hline primary & -0.4637706 & 0.0458781 & -10.11 & 0.000 & $-.55369-.3738512$ \\
\hline secondary & -0.9691338 & 0.3312863 & -2.93 & 0.003 & $-1.618443 \quad-.3198247$ \\
\hline higher & -1.6755 & 0.7211637 & -2.32 & 0.02 & $-3.088955 \quad-.2620451$ \\
\hline \multicolumn{6}{|l|}{ Wealth index, poor(ref.) } \\
\hline medium & -0.0869593 & 0.0434646 & -2.00 & 0.045 & $-.1721482-.0017703$ \\
\hline rich & -0.0910793 & 0.0423884 & -2.15 & 0.032 & $-.174159-.0079995$ \\
\hline \multicolumn{6}{|l|}{$\begin{array}{l}\text { Mother age at the first } \\
\text { birth, }<15 \text { (ref.) }\end{array}$} \\
\hline $15-19$ & -0.4489802 & 0.0492739 & -9.11 & 0.000 & $-.5455553 \quad-.352405$ \\
\hline 20 and above & -0.6945656 & 0.0542082 & -12.81 & 0.000 & $-.8008118 \quad-.5883194$ \\
\hline \multicolumn{6}{|c|}{$\begin{array}{l}\text { Currently breastfeeding, } \\
\text { no(ref.) }\end{array}$} \\
\hline yes & -0.2534029 & 0.0347195 & -7.3 & 0.000 & $-.3214518 \quad-.185354$ \\
\hline \multicolumn{6}{|l|}{$\begin{array}{l}\text { Current mother working, } \\
\text { no(ref.) }\end{array}$} \\
\hline yes & 0.0755776 & 0.0364916 & 2.07 & 0.038 & .0040554 . 1470999 \\
\hline _cons & -0.4335085 & 0.0812158 & -5.34 & 0.000 & $-.5926885-.2743285$ \\
\hline $\log ($ children ever born) & 1.000 & & & & \\
\hline \multicolumn{6}{|l|}{ inflate } \\
\hline \multicolumn{6}{|l|}{$\begin{array}{l}\text { Marital status, } \\
\text { married(ref.) }\end{array}$} \\
\hline Unmarried & 1.03762 & 0.2837085 & 3.66 & 0.000 & $.4815612 \quad 1.593678$ \\
\hline cons & -1.959543 & 0.3535019 & -5.54 & 0.000 & $-2.652394-1.266692$ \\
\hline /Inalpha & -1.274886 & 0.2225431 & -5.73 & 0.000 & $-1.711062-.8387092$ \\
\hline alpha & 0.2794629 & 0.0621925 & & & $\begin{array}{ll}.1806738 & .4322681\end{array}$ \\
\hline
\end{tabular}

ref. $=$ reference category of the variable. 
Figures

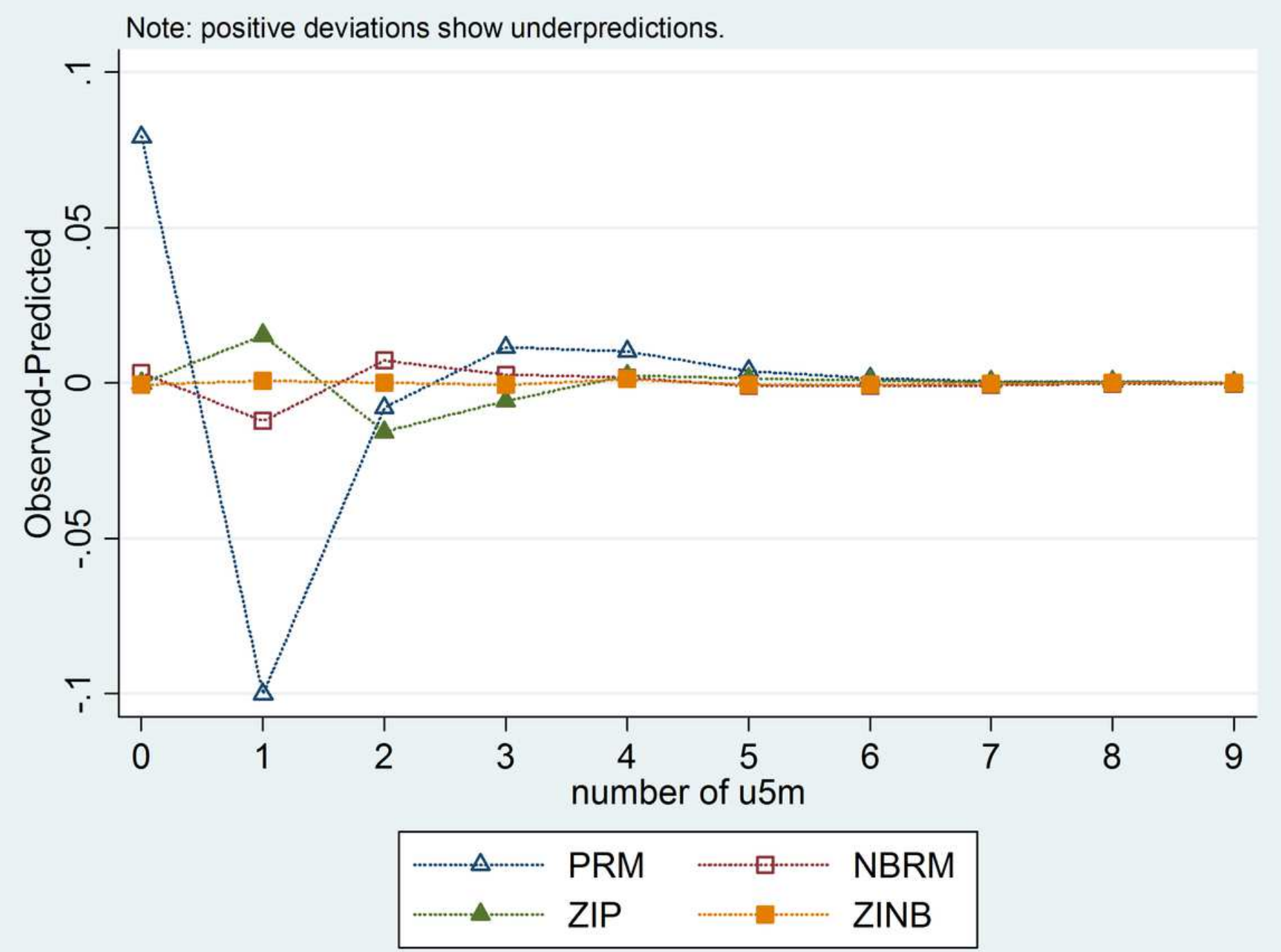

Figure 1

Residual plots for estimated models 